\title{
Anti-high-mobility group box-1 (HMGB1) mediates the apoptosis of alveolar epithelial cells (AEC) by receptor of advanced glycation end-products (RAGE)/c-Jun N-terminal kinase (JNK) pathway in the rats of crush injuries
}

Bin-Fei Zhang, Wei Song, Jun Wang, Peng-Fei Wen and Yu-Min Zhang*

\begin{abstract}
Objectives: The lung injury is often secondary to severe trauma. In the model of crush syndrome, there may be secondary lung injury. We hypothesize that high-mobility group box 1 (HMGB1), released from muscle tissue, mediates the apoptosis of alveolar epithelial cells (AEC) via HMGB1/Receptor of advanced glycation end-products (RAGE)/c-Jun $\mathrm{N}$-terminal kinase (JNK) pathway. The study aimed to investigate how HMGB1 mediated the apoptosis of AEC in the rat model.

Methods: Seventy-five SD male rats were randomly divided into five groups: CS, CS + vehicle, CS + Ethyl pyruvate (EP), CS + FPS-ZM1 group, and CS + SP600125 groups. When the rats CS model were completed after $24 \mathrm{~h}$, the rats were sacrificed. We collected the serum and the whole lung tissues. Inflammatory cytokines were measured in serum samples. Western blot and RT-qPCR were used to quantify the protein and mRNA. Lastly, apoptotic cells were detected by TUNEL. We used SPSS 25.0 for statistical analyses.

Results: Nine rats died during the experiments. Dead rats were excluded from further analysis. Compared to the CS group, levels of HMGB1 and inflammatory cytokines in serum were downregulated in CS + EP, CS + FPS-ZM1, and CS + SP600125 groups. Western blot and RT-qPCR analysis revealed a significant downregulation of HMGB1, RAGE, and phosphorylated-JNK in CS + EP, CS + FPS-ZM1, and CS + SP600125 groups, compared with the CS groups, excluding total-JNK mRNA. Apoptosis of AEC was used TUNEL to assess. We found the TUNEL-positive cells were downregulated in CS + EP, CS + FPS-ZM1, and CS + SP600125 groups.
\end{abstract}

Conclusion: The remote lung injury begins early after crush injuries. The HMGB1/RAGE/JNK signaling axis is an attractive target to abrogate the apoptosis of AEC after crush injuries.

Keywords: Crush injury, High-mobility group box-1, Receptor of advanced glycation end-products (RAGE), c-Jun N-terminal kinase (JNK), SP600125, Apoptosis, Alveolar epithelial cells

*Correspondence: zym2666@126.com

Department of Joint Surgery, Honghui Hospital, Xi'an Jiaotong University, No. 555 Youyi East Road, Beilin District, Xi'an, Shaanxi Province 710054,

People's Republic of China

\section{Introduction}

Crush injuries are severe soft tissues damage in the field of orthopedic trauma. Prolonged compression of limb muscles and subsequent decompression is essential in the development of crush syndrome (CS) [1], including original author(s) and the source, provide a link to the Creative Commons licence, and indicate if changes were made. The images or other third party material in this article are included in the article's Creative Commons licence, unless indicated otherwise in a credit line to the material. If material is not included in the article's Creative Commons licence and your intended use is not permitted by statutory regulation or exceeds the permitted use, you will need to obtain permission directly from the copyright holder. To view a copy of this licence, visit http://creativecommons.org/licenses/by/4.0/. The Creative Commons Public Domain Dedication waiver (http://creativeco mmons.org/publicdomain/zero/1.0/) applies to the data made available in this article, unless otherwise stated in a credit line to the data. 
hyperkalemia, metabolic acidosis, hypovolemic shock, acute kidney injury, and disseminated intravascular coagulation [2]. The sequential damage in the kidney after crush injury is not the unique dysfunctional organ [3]. It has been proved that the secondary damage in the lung [4-6], heart [7], and liver [8] contributes to multiple organs failure [9]. In fact, the pathophysiology following CS is a procedure of systemic inflammatory introducing injuries.

The lung injury has been observed clinically after severe trauma. This lung injury is secondary to the trauma, and likely due to an elevation in leukocytes and inflammatory cytokines in the lung tissue [10]. Duration of the treatment of trauma and the lung injury is often paid attention to when finding worse pulmonary function. It may be that the lung injury begins at the time of decompression. Therefore, we should notice that the remote lung injury at the early stage.

Currently, numerous studies have focused on a proinflammatory mediator, high-mobility group box 1 (HMGB1) [11-13]. In the model of CS, the levels of HMGB1 in muscle increased to peak at $12 \mathrm{~h}$ after decompression in the mice model [14]. In addition, the administration of anti-HMGB1 antibody improves survival rate and suppresses serum levels of HMGB1 and inflammation cytokines [6]. Significantly, the treatment for HMGB1 could ameliorate lung injury $[15,16]$.

However, the relationship between extracellular HMGB1 and lung injury is uncertain in crush injuries. The previous study has shown that HMGB1 is released from muscle tissue in response to damage immediately, and the level of serum HMGB1 increases quickly after decompression in the rats model [6]. The HMGB1 can be passively released from damaged cells as a proinflammatory alarmin [17]. When released HMGB1 enters the systemic circulation, the sequential response in remote lung injury follows. The receptor of advanced glycation end-products (RAGE) is a cell surface transmembrane multiligand receptor, and it can bind a large variety of endogenous ligands, such as HMGB1, leading to its classification as a pattern recognition receptor [18]. In the LPS-induced lung injury, the injury was found via HMGB1-RAGE signaling in alveolar epithelial cells (AEC). In addition, c-Jun N-terminal kinase (JNK) is an essential transducing enzyme that is involved in apoptosis of AEC [19], and JNK inhibitor SP600125 could improve cell apoptosis in acute lung injury [20]. The RAGE/JNK pathway has also been proved in participating the inflammation response [21].

Thus, we hypothesize that HMGB1, released from muscle tissue, mediates the apoptosis of AEC via the HMGB1/RAGE/JNK pathway. This study aimed to investigate the hypothesis via different inhibitors in the rats model.

\section{Materials and methods \\ Animals}

Seventy-five SD male rats weighing 250-300 g were purchased from the Laboratory Animal Center of Xian Jiaotong University. All animal procedures followed the ARRIVE guidelines [22] and the National Institutes of Health guide for the care and use of laboratory animals [23]. The Ethics Committee approved the study of Honghui Hospital, Xi'an Jiaotong University (2018025).

\section{Treatment}

The rats were housed and fed in a temperature of $22 \pm 1{ }^{\circ} \mathrm{C}$ and humidity $45-55 \%$ environment with a standardized light-dark cycle ( $12 \mathrm{~h}$ day/night), and they were free to eat and drink. After one week of adaptive feeding, they were randomly divided into five groups: CS group, CS + vehicle group, CS + Ethyl pyruvate (EP) group, CS + FPS-ZM1 group, and CS + SP600125 group. The concentration of ethyl pyruvate, FPS-ZM1, and $S P 600125$ was $50 \mathrm{mg} / \mathrm{kg}, 5 \mathrm{mg} / \mathrm{kg}$, and $15 \mathrm{mg} / \mathrm{kg}$, respectively.

We injected the above drugs into the intraperitoneal cavity before compression $0.5 \mathrm{~h}$ and used DMSO as the vehicle. (The injection volume was $0.7 \mathrm{~mL}$.) CS + vehicle group was injected with the same volume of DMSO as a negative control, and the CS group was used as a blank control.

\section{Rats model of CS}

After administering inhibitors $0.5 \mathrm{~h}$, the SD rats were anesthetized by intraperitoneal injection of 3\% sodium pentobarbital $(30 \mathrm{mg} / \mathrm{kg})$. After anesthesia, the rats were fixed in the prone position, and the hind limbs were compressed at $4.5 \mathrm{~cm}$ away from the ankles, with $20 \mathrm{~kg}$ [24] heavy for $3 \mathrm{~h}$. Because the mortality was high when compressing $6 \mathrm{~h}$ in a preliminary study, we chose to compress $3 \mathrm{~h}$. The body temperature of the rats was maintained at $37 \pm 0.5{ }^{\circ} \mathrm{C}$ during this period. After the compression was removed, the rats were allowed to recover for $24 \mathrm{~h}$. When the recovery time was up to $24 \mathrm{~h}$, the blood of rats was collected, the serum was separated, and then the rats were sacrificed. We collected the whole lung tissue and divided each lung tissue into two parts: one part of the lung tissue was stored in liquid nitrogen, and the other part of the lung tissue was fixed with $4 \%$ paraformaldehyde solution for later use.

\section{ELISA}

All serum samples were tested for HMGB1 (FineTest, Wuhan, China), tumor necrosis factor- $\alpha$ (TNF- $\alpha$ ) 
(Wanleibio, Shenyang, China), IL-1 $\beta$ (Wanleibio, Shenyang, China), and IL-6 (Wanleibio, Shenyang, China), using a rat ELISA kit. The ELISA assay was performed following the manufacturer's protocols. The optical density of each well was measured spectrophotometrically at $450 \mathrm{~nm}$ within 5 min after adding the stop solution using a microplate reader (ELX-800, BIOTEK, USA).

\section{Western blot}

The frozen tissue samples were solubilized in RIPA buffer on ice using a homogenizer. Samples $(50 \mu \mathrm{g} / \mathrm{lane})$ were separated on a sodium dodecyl sulfate-polyacrylamide gel $12 \%$ gel) and electrotransferred onto polyvinylidene fluoride membranes. After $2 \mathrm{~h}$ incubation in blocking solution (5\% non-fat milk in $20 \mathrm{mM}$ Tris- $\mathrm{HCl}, 150 \mathrm{mM}$ $\mathrm{NaCl}, 0.1 \%$ Tween-20; TBST), the membranes were blotted with primary antibodies against HMGB1 (1:1000, A19529, ABclonal), RAGE (1:1000, AF5309, Affinity), p-JNK (1:500, WL01295, Wanleibio), JNK (1:500, WL01813, Wanleibio), or $\beta$-actin (1:500, Wanleibio) overnight at $4{ }^{\circ} \mathrm{C}$. After extensive rinsing with TBST buffer, the blots were incubated with HRP-conjugated secondary antibodies (Abgent, San Diego, CA, USA). The density of the bands was analyzed by Quantity One software version 4.62 (Bio-Rad, USA).

\section{Real-time qPCR}

Total RNA from the rats' lung tissues was extracted by TRIpure reagent (RP1001, BioTeke, China). A commercial reverse transcription system BeyoRT II M-MLV (D7160L, Beyotime Biotechnology, China) was used to synthesize cDNA from purified total RNA. qRT-PCR was conducted using SYBR Green (SY1020, Solarbio, China) on a thermocycler (Exicycler ${ }^{\mathrm{TM}}$ 96, BIONEER, Korea). The sequences of primers used are listed in Table 1. $\beta$-actin was used as an internal control for indicated genes. The relative expression of a target gene was determined by the $2^{-\triangle \triangle \mathrm{CT}}$ method (NANO 2000, Thermo, USA).

\section{TUNEL staining}

We used a terminal deoxynucleotidyl transferase-mediated dUTP nick end-labeling (TUNEL) reaction utilizing TUNEL Kit (WLA127a, Wanleibio, China) to detect apoptotic cells. The tissue sections were deparaffinized with xylene and treated with $\mathrm{H} 2 \mathrm{O} 2$ to inactivate endogenous peroxidase. After washing with PBS, the following operations were completed in a dark room. The specimens were incubated in the red labeling reaction mixture containing terminal deoxynucleotidyl transferase. The sections were then counterstained with a blue DAPI solution (D106471, Aladdin-e, China). Finally, the fluorescence images were collected in a camera system (DP73, OLYMPUS, Japan).

\section{Statistical analysis}

SPSS 25.0 (SPSS Inc., Chicago, IL, USA) was used for statistical analyses. All data were presented as mean \pm SD. Comparisons among multiple groups were performed using ANOVA. Comparisons between the two groups were performed using the LSD test. $P<0.05$ was considered statistically significant.

\section{Results}

\section{Mortality rate and general observations}

In total, nine rats died after the compression and administration of drugs during the experiments. The number of deaths was 2, 3, 1, 2, and 1 in CS, CS + vehicle, CS + EP, CS + FPS-ZM1, and CS + SP600125 groups, respectively. There was no significant difference in mortality rate $(P=0.778)$. Dead rats were excluded from further analysis. After the modeling, activities of rats reduced obviously, with both hind limbs prolapsing. In all living rats, we found injured skeletal muscles to be hemorrhagic and with edema.

Table 1 Sequences of primers $\left(5^{\prime}-3^{\prime}\right)$

\begin{tabular}{lllll}
\hline Primer & Sequence & Primer length & Tm & Product length \\
\hline HMGB1 F & TGACAAGGCTCGTTATGAAAG & 21 & 55.8 & 186 \\
HMGB1 R & TTCTTCGCAACATCACCAAT & 20 & 56.2 & 64.4 \\
RAGE F & GACGGGACTCTTCACGCTTCG & 21 & 61.8 & 192 \\
RAGE R & CCACCTTCAGGCTCAACCAAC & 21 & 54.7 & 161 \\
JNKF & GATTTGGAGGAGCGAACTAA & 20 & 58 & 155 \\
JNKR & CTGCTGTCTGTATCCGAGGC & 20 & 60.1 & 62 \\
$\beta$-actin F & GGAGATTACTGCCCTGGCTCCTAGC & 25 & 25 & \\
$\beta$-actin R & GGCCGGACTCATCGTACTCCTGCTT & 25 & & 16 \\
\hline
\end{tabular}


The levels of HMGB1 and inflammatory cytokines in serum When testing the levels of serum HMGB1, we found that there was no significant statistical difference between the CS group and CS + vehicle group. Compared to the CS group, the level of HMGB1 was downregulated in CS + EP, CS + FPS-ZM1, and CS + SP600125 groups (Fig. 1a).

As for the inflammatory cytokines of TNF- $\alpha$, IL-1 $\beta$, and IL-6, there was no significant difference between the $\mathrm{CS}$ and $\mathrm{CS}+$ vehicle groups. However, the levels of these cytokines were lower in CS + EP, CS + FPS-ZM1, and CS + SP600125 groups, compared to the CS group (Fig. 1b-d).
Effect of EP, FPS-ZM1, and SP600125 on the protein expression of HMGB1, RAGE, and JNK

Western blot analysis revealed a significant downregulation of HMGB1 in CS + EP, CS + FPS-ZM1, and $\mathrm{CS}+\mathrm{SP} 600125$ groups, compared with the CS groups (Fig. 2a). As for the RAGE and p-JNK, there was also significant downregulation in CS + EP, CS + FPS-ZM1, and $\mathrm{CS}+\mathrm{SP600125}$ groups (Fig. 2b, c).

\section{Effect of EP, FPS-ZM1, and SP600125 on the mRNA} expression of HMGB1, RAGE, and JNK

RT-qPCR analysis revealed a significant downregulation of HMGB1 and RAGE mRNA in CS+EP, CS+FPSZM1, and CS + SP600125 groups, compared with the CS

\section{a}

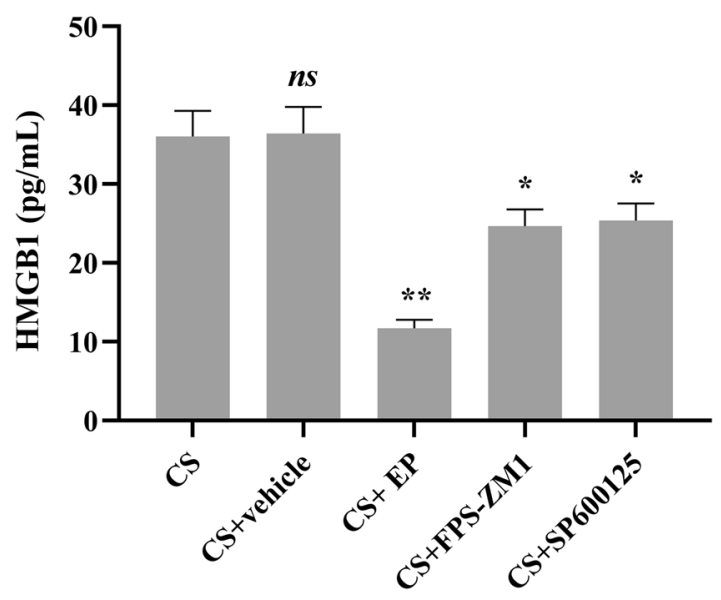

c

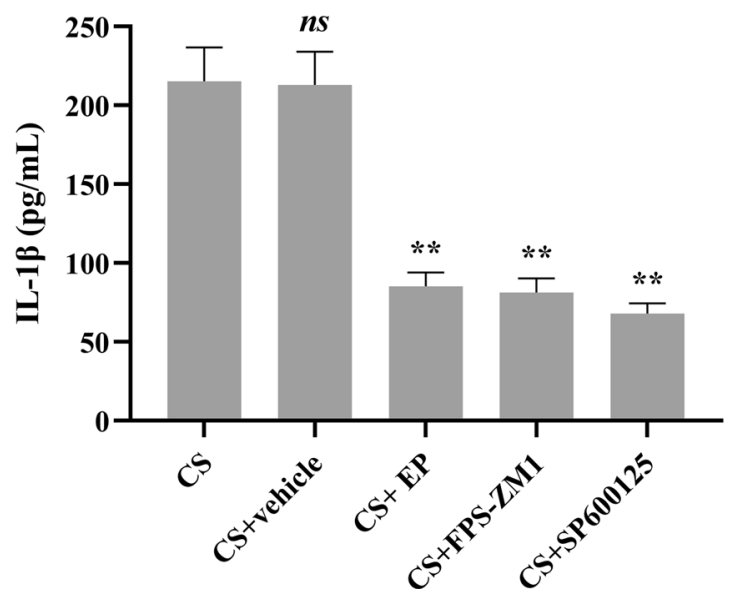

b

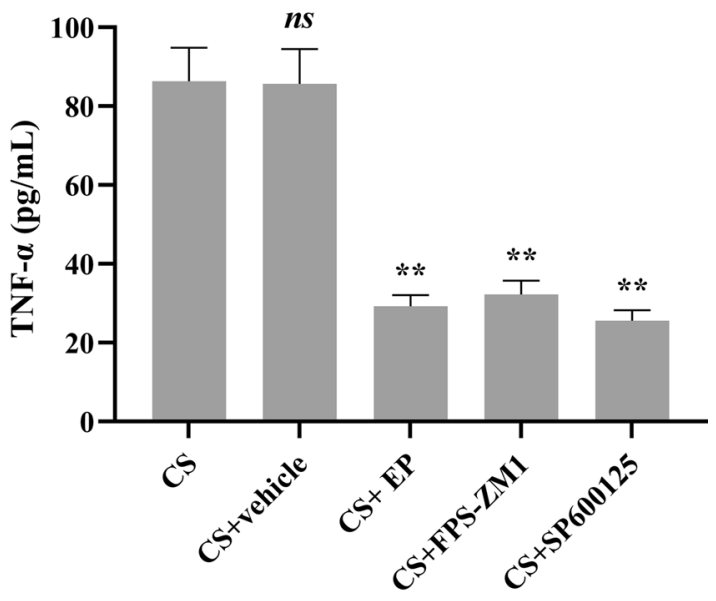

d

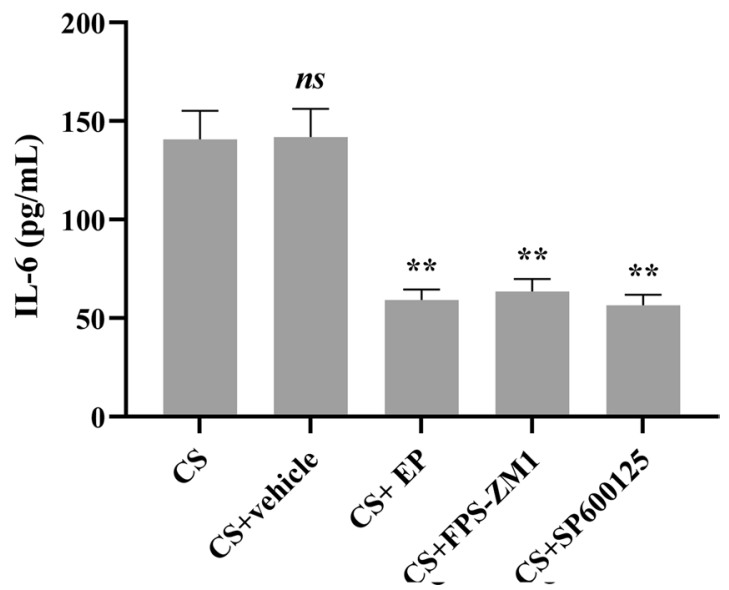

Fig. 1 EP, FPS-ZM1, and SP600125 affect the expression of HMGB1 and inflammatory cytokines in serum. Relative levels of HMGB1,TNF-a, IL-1 $\beta$, and IL-6 were analyzed using ELISA kits. The HMGB1 and all inflammatory cytokines were lower in the later three pretreatment groups. Results were expressed as means \pm SDs. The number of rats were 13, 12, 14, 13, and 14 in CS, CS + vehicle, CS + EP, CS + FPS-ZM1, and CS + SP600125 groups, respectively. Statistical significance: $n s P \geq 0.05,{ }^{*} P<0.05$ and ${ }^{*} P<0.01$, versus CS group 

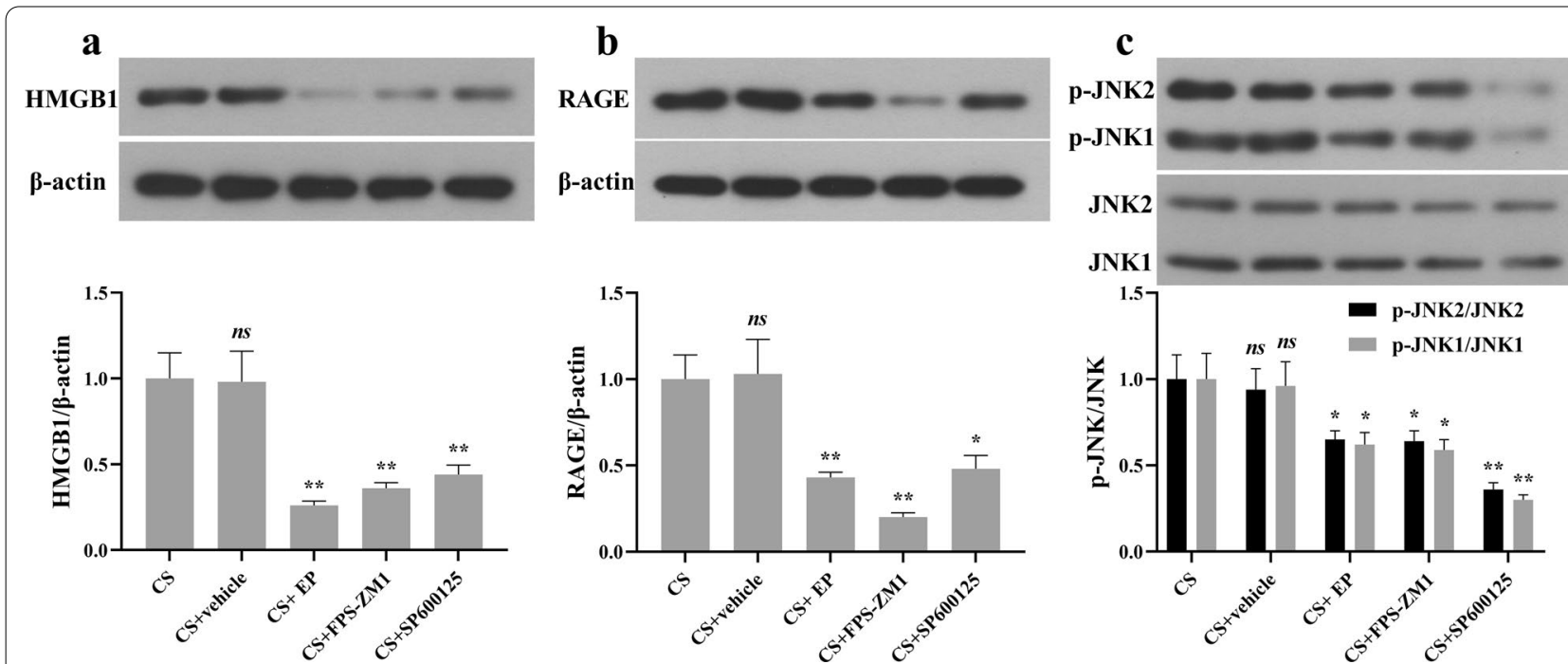

Fig. 2 EP, FPS-ZM1, and SP600125 affect the expression of HMGB1, RAGE, and JNK in lung tissue. Relative levels of HMGB1, RAGE, and JNK were analyzed using Western blot. The HMGB1, RAGE, and p-JNK were lower in the later three pretreatment groups. Results were expressed as means $\pm S D$ s. The number of rats were 13,12, 14, 13, and 14 in CS, CS + vehicle, CS + EP, CS + FPS-ZM1, and CS + SP600125 groups, respectively. Statistical significance: $n s P \geq 0.05,{ }^{*} P<0.05$ and ${ }^{* *} P<0.01$, versus CS group

groups (Fig. 3a, b). As for the mRNA expression of JNK, there was no difference in the five groups (Fig. 3c).

\section{Apoptosis of AEC}

We used TUNEL to assess the apoptosis of AEC. As shown in Fig. 4, most of the AEC were TUNEL-positive cells in CS and CS + vehicle groups, and the number of positive cells decreased in the other three groups. After the statistic, we found there was no significant difference between the CS and CS + vehicle groups $(P>0.05)$ and TUNEL-positive cells in CS +EP, CS + FPS-ZM1, and CS + SP600125 groups were lower than the CS group $(P<0.05)$.

\section{Discussion}

In clinical, the lung injury maybe start at the early stage after crush injuries. The surgeons should notice the dynamic changes in pulmonary function. There were two main reasons we designed this study. On the one hand, some animals had the amount of pink foam in the mouth and nose when making animal models. We highly suspect the occurrence of secondary lung injury in the crush injury. On the other hand, this signaling pathway has not been verified in CS animal models. Thus, we conducted this animal experiment. In this study, we explored the potential role of the HMGB1/RAGE/JNK pathway on apoptosis of AEC to show the damage to lung tissue under the model of crush injuries. Our results indicate the following main findings. Firstly, the HMGB1 in muscle is rapidly released to the systemic circulation, and the remote lung injury begins early after crush injuries. Secondly, the HMGB1/RAGE/JNK pathway is involved in the pathophysiology of lung injury following CS. Thirdly, inhibitors of HMGB1, RAGE, and JNK could alleviate the apoptosis of AEC and serum inflammatory cytokines.

In terms of animal modeling, we chose SD rats and compression for $3 \mathrm{~h}$. There are two reasons. The first reason was the degree of muscle damage at $3 \mathrm{~h}$ was the same as those at $6 \mathrm{~h}$ in the preliminary experiment, but there were more necrotic cells at $6 \mathrm{~h}$. The second reason was that the mortality rate of rats at $6 \mathrm{~h}$ was too high, nearly $70-80 \%$. It meant requiring too many experimental animals. In particular, the survival animals may have the possibility of selection bias, which would affect the reliability of the experimental results.

As for the HMGB1, RAGE, and JNK signaling inhibitors, we have chosen EP, FPS-ZM1, and SP600125 for intervention. The role of EP was chelating $\mathrm{Ca}^{2+}$ directly and responsible for the suppression of HMGB1 [25]. EP has been used to abrogate the pathological effects of HMGB1 [25, 26]. FPS-ZM1 is a blood-brain-barrier permeant, tertiary amide compound which is a highaffinity RAGE-specific inhibitor, blocking $A \beta$ binding to the V domain of RAGE [27]. FPS-ZM1 was often used to inhibit the role of RAGE in an animal experiment [28, 29]. Moreover, an inhibitor of JNK, SP600125 [20], has been widely used. Thus, these administrations were specific blocking drugs to the signaling axis. We chose to give the drugs into the intraperitoneal cavity before compression to ensure they completely play the role. 


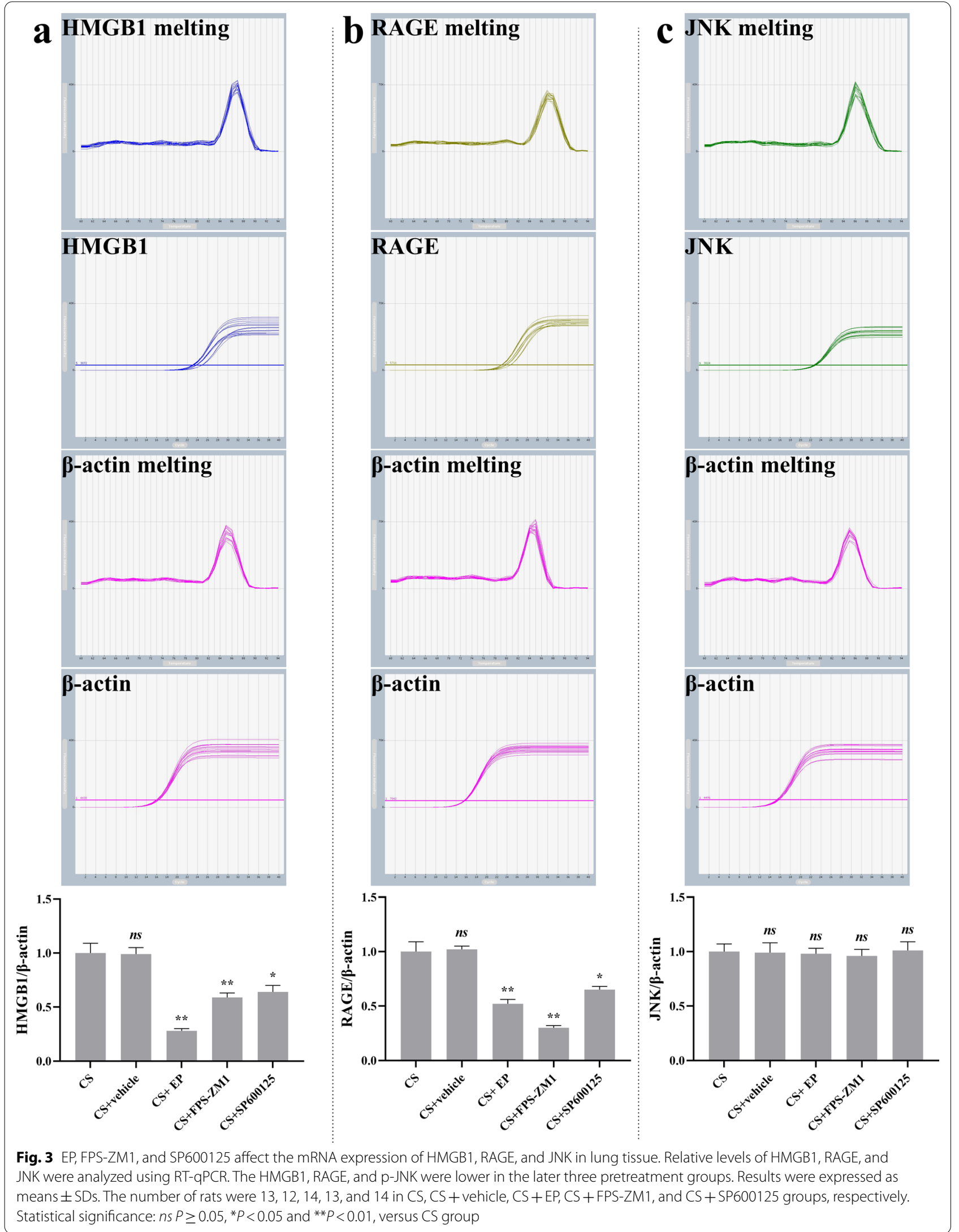




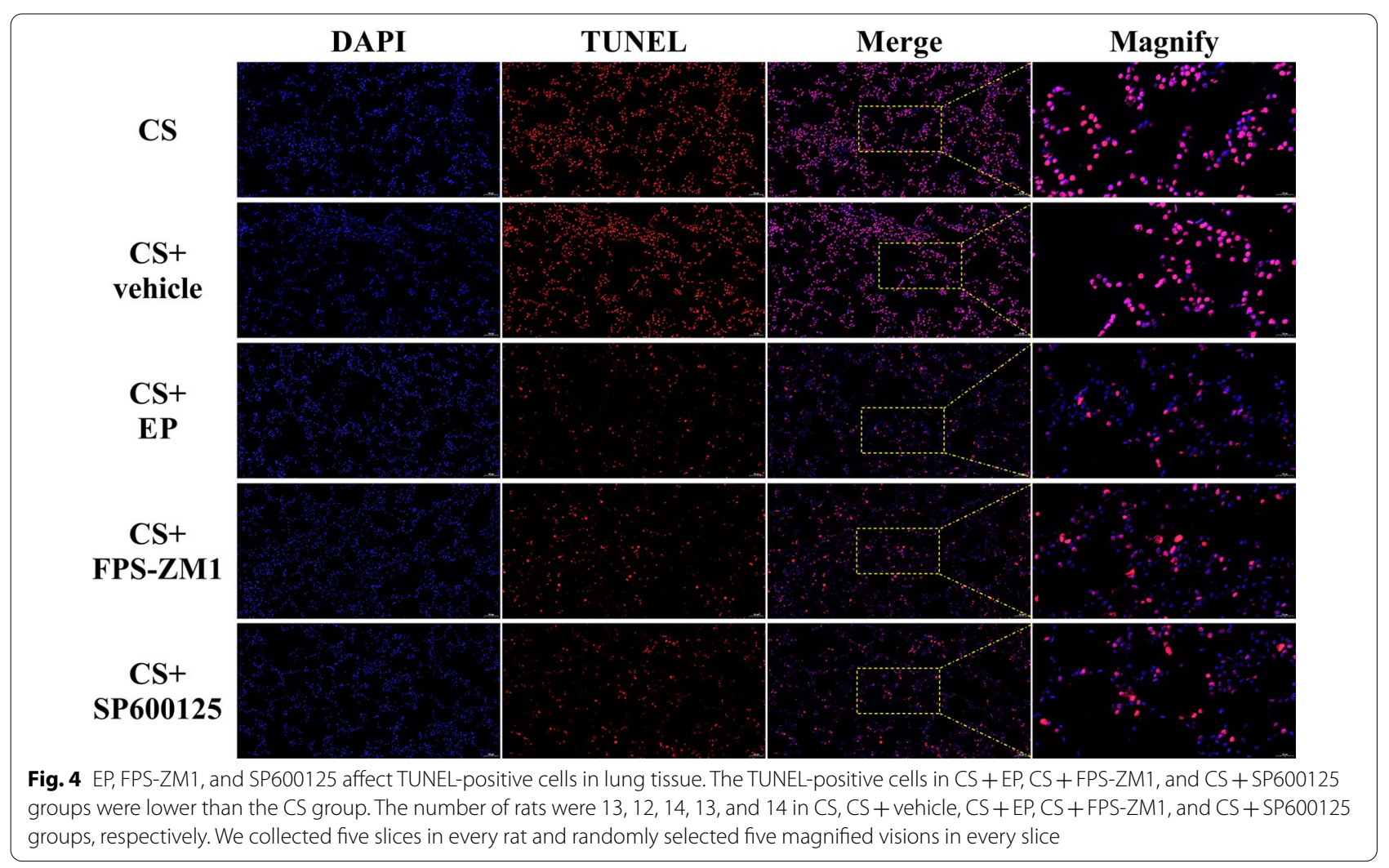

In terms of detection methods, we adopted western blot and RT-qPCR techniques for mechanism exploration. Since the protein and mRNA expression trends were similar, the experimental results were relatively reliable. In addition, TUNEL was used to detect apoptosis of AEC [30]. Thus, these methods were typical and reliable.

The HMGB1/RAGE/JNK pathway has been studied in some studies, and the role of this pathway has been established in dopaminergic neurons [31], degenerated human nucleus pulposus cells [32]. In this study, we have identified the signaling axis of HMGB1/RAGE/JNK in lung injury after CS. The HMGB1 in muscle was released to the systemic circulation. When HMGB1 arrived at the lung, the RAGE/JNK pathway in AEC was activated, and the pathophysiology of lung injury began. Lastly, apoptosis becomes one of the damaged types of AEC. Furthermore, the treatment of inhibiting or blocking the role of HMGB1 may moderate the lung injury.

However, there are some limitations to this study. Firstly, we could not confirm whether inflammatory cytokines are released from damaged muscle cells or a response from other cells. Secondly, it is uncertain whether the apoptotic cell type is AEC type I or II or both. The above shortcomings need to be further implemented in cell research.

\section{Conclusions}

The remote lung injury begins early after crush injuries. The HMGB1/RAGE/JNK signaling axis is an attractive target to abrogate the apoptosis of AEC after crush injuries.

\section{Abbreviations}

CS: Crush syndrome; HMGB1: High-mobility group box-1 protein; RAGE: Receptor of advanced glycation end-products; JNK: C-Jun N-terminal kinase; AEC: Alveolar epithelial cells; TNF-a: Tumor necrosis factor-a; TUNEL: Terminal deoxynucleotidyl transferase-mediated dUTP nick end-labeling.

\section{Acknowledgements}

Not applicable.

\section{Authors' contributions}

$\mathrm{B}-\mathrm{F} Z$ and $\mathrm{Y}-\mathrm{M} Z \mathrm{Z}$ conceived and designed the experiments. B-F Z,W S, JW, and P-FW performed the experiments. B-F Z analyzed the data. B-F Z wrote the manuscript. All authors read and approved the final manuscript.

\section{Funding}

This work was supported by the Natural Science Foundation of Shaanxi Province (Grant numbers 2020JQ-961).

Availability of data and material Please contact author for data requests. 


\section{Declarations}

Ethics approval and consent to participate

The study was approved by the Ethics Committee of Honghui Hospital, Xi'an Jiaotong University (No. 2018025).

\section{Consent to publish}

The work described has not been published before (except in the form of an abstract or as part of a published lecture, review, or thesis); it is not under consideration for publication elsewhere; and its publication has been approved by all co-authors.

\section{Competing interests}

The authors have declared that no competing interests exist.

Received: 2 November 2021 Accepted: 30 December 2021

Published online: 15 January 2022

\section{References}

1. Gonzalez D. Crush syndrome. Crit Care Med. 2005;33(1 Suppl):S34-41.

2. Malinoski D, Slater M, Mullins R. Crush injury and rhabdomyolysis. Crit Care Clin. 2004:20(1):171-92

3. Lv Q, Long $\mathrm{M}$, Wang $\mathrm{X}$, et al. The role of alpha-1-acid glycoprotein in the diagnosis and treatment of crush syndrome-induced acute kidney injury. Shock. 2021;56:1028.

4. Sonoi $\mathrm{H}$, Matsumoto $\mathrm{N}$, Ogura $\mathrm{H}$, et al. The effect of antithrombin on pulmonary endothelial damage induced by crush injury. Shock. 2009;32(6):593-600

5. Nakagawa J, Matsumoto N, Nakane Y, et al. The Beneficial effects of ETSGS, a novel Vitamin E derivative, on a rat model of crush injury. Shock. 2016:46(6):681-7.

6. Shimazaki J, Matsumoto N, Ogura H, et al. Systemic involvement of highmobility group box 1 protein and therapeutic effect of anti-high-mobility group box 1 protein antibody in a rat model of crush injury. Shock. 2012;37(6):634-8

7. Liu S, Yu Y, Luo B, et al. Impact of traumatic muscle crush injury as a cause of cardiomyocyte-specific injury: an experimental study. Heart Lung Circ. 2013;22(4):284-90.

8. Sgarbi M, Silva B, Peres C, et al. Leukocyte infiltration in lung, muscle, and liver after limb compression in rats. Pathophysiology. 2013;20(2):111-6.

9. Xu P, Wang F, Zhou X, et al. Systemic inflammatory response and multiple organ dysfunctions following crush injury: a new experimental model in rabbits. Inflammation. 2018;41(1):240-8.

10. Wu X, Schwacha MG, Dubick MA, et al. Trauma-related acute lung injury develops rapidly irrespective of resuscitation strategy in the rat. Shock. 2016:46(3 Suppl 1):108-14.

11. Yamamoto T, Ono T, Ito T, et al. Hemoperfusion with a high-mobility group box 1 adsorption column can prevent the occurrence of hepatic ischemia-reperfusion injury in rats. Crit Care Med. 2010;38(3):879-85.

12. Kang R, Zhang Q, Hou W, et al. Intracellular Hmgb1 inhibits inflammatory nucleosome release and limits acute pancreatitis in mice. Gastroenterology. 2014;146(4):1097-107.

13. Andersson U, Ottestad W, Tracey K. Extracellular HMGB1: a therapeutic target in severe pulmonary inflammation including COVID-19? Mol Med. 2020;26(1):42

14. Zhang $B$, Wang $P$, Cong Y, et al. Anti-high mobility group box-1 (HMGB1) antibody attenuates kidney damage following experimental crush injury and the possible role of the tumor necrosis factor-a and c-Jun N-terminal kinase pathway. J Orthop Surg Res. 2017;12(1):110.

15. Wang M, Gauthier A, Daley L, et al. The role of HMGB1, a nuclear damageassociated molecular pattern molecule, in the pathogenesis of lung diseases. Antioxid Redox Signal. 2019;31(13):954-93.

16. Qu L, Chen C, Chen Y, et al. High-mobility Group Box 1 (HMGB1) and autophagy in acute lung injury (ALI): a review. Med Sci Monit. 2019;25:1828-37.

17. Scaffidi P, Misteli T, Bianchi M. Release of chromatin protein HMGB1 by necrotic cells triggers inflammation. Nature. 2002;418(6894):191-5.
18. Oczypok E, Perkins T, Oury T. All the "RAGE" in lung disease: the receptor for advanced glycation endproducts (RAGE) is a major mediator of pulmonary inflammatory responses. Paediatr Respir Rev. 2017;23:40-9.

19. Fu Y, Fang F, Lu Z, et al. N-acetylcysteine protects alveolar epithelial cells from hydrogen peroxide-induced apoptosis through scavenging reactive oxygen species and suppressing c-Jun $\mathrm{N}$-terminal kinase. Exp Lung Res. 2010;36(6):352-61.

20. Shen H, Wu N, Wang Y, et al. JNK inhibitor SP600125 attenuates paraquatinduced acute lung injury: an in vivo and in vitro study. Inflammation. 2017:40(4):1319-30

21. Wu X, Mi Y, Yang H, et al. The activation of HMGB1 as a progression factor on inflammation response in normal human bronchial epithelial cells through RAGE/JNK/NF-KB pathway. Mol Cell Biochem. 2013;380:249-57.

22. Percie du Sert N, Ahluwalia A, Alam S, et al. Reporting animal research: Explanation and elaboration for the ARRIVE guidelines 2.0. PLoS Biol. 2020;18(7):e3000411.

23. Council NR. Guide for the care and use of laboratory animals. Washington, DC: The National Academies Press; 1996.

24. Fan BS, Zhang EH, Wu M, et al. Activation of alpha7 nicotinic acetylcholine receptor decreases on-site mortality in crush syndrome through insulin signaling-Na/K-ATPase pathway. Front Pharmacol. 2016;7:79.

25. Shin J, Kim I, Kim S, et al. Ethyl pyruvate inhibits HMGB1 phosphorylation and release by chelating calcium. Mol Med. 2015;20:649-57.

26. Bhat $S$, Massey N, Karriker L, et al. Ethyl pyruvate reduces organic dustinduced airway inflammation by targeting HMGB1-RAGE signaling. Respir Res. 2019;20(1):27.

27. Zhou H, Jin C, Cui L, et al. HMGB1 contributes to the irradiation-induced endothelial barrier injury through receptor for advanced glycation endproducts (RAGE). J Cell Physiol. 2018;233(9):6714-21.

28. Liu Y, Shen W, Chen Q, et al. Inhibition of RAGE by FPS-ZM1 alleviates renal injury in spontaneously hypertensive rats. Eur J Pharmacol. 2020:882:173228

29. Deane R, Singh I, Sagare AP, et al. A multimodal RAGE-specific inhibitor reduces amyloid beta-mediated brain disorder in a mouse model of Alzheimer disease. J Clin Invest. 2012;122(4):1377-92.

30. Li J, Zhou Q, Liang Y, et al. miR-486 inhibits PM2.5-induced apoptosis and oxidative stress in human lung alveolar epithelial A549 cells. Ann Transl Med. 2018:6(11):209.

31. Kim S, Ryu M, Han J, et al. Activation of the HMGB1-RAGE axis upregulates TH expression in dopaminergic neurons via JNK phosphorylation. Biochem Biophys Res Commun. 2017;493(1):358-64.

32. Niu C, Lin S, Yuan L, et al. Upregulation of miR-107 expression following hyperbaric oxygen treatment suppresses HMGB1/RAGE signaling in degenerated human nucleus pulposus cells. Arthritis Res Ther. 2019;21(1):42

\section{Publisher's Note}

Springer Nature remains neutral with regard to jurisdictional claims in published maps and institutional affiliations.

Ready to submit your research? Choose BMC and benefit from:

- fast, convenient online submission

- thorough peer review by experienced researchers in your field

- rapid publication on acceptance

- support for research data, including large and complex data types

- gold Open Access which fosters wider collaboration and increased citations

- maximum visibility for your research: over 100M website views per year

At BMC, research is always in progress.

Learn more biomedcentral.com/submissions 